\title{
A New Multi-Agent Approach to Adaptive E-Education
}

\author{
Jing Chen and Peng Cheng \\ Department Of Computer Science, Huazhong Normal University, Wuhan, \\ 430079, P.R.China \\ dancinglulu@sina.com
}

\begin{abstract}
Improving customer satisfaction degree is important in e-Education. This paper describes a new approach to adaptive e-Education taking into account the full spectrum of Web service techniques and activities. It presents a multi-agents architecture based on artificial psychology techniques, which makes the e-Education process both adaptable and dynamic, and hence up-todate. Knowledge base techniques are used to support the e-Education process, and artificial psychology techniques to deal with user psychology, which makes the e-Education system more effective and satisfying.
\end{abstract}

\section{Introduction}

In the age of the new information and communication technology, it should be possible to learn not only by a locally available electronic support, i.e. an interactive CD-Rom, but even "far away" from the teaching source. One challenge for designers and $\mathrm{HCI}$ researchers is to develop software tools able to engage novice learners and to support their learning even at distance.

Usability is the vital role of e-Education systems [1]. But there are more works can be done. Customer satisfaction is going to be more and more important to eEducation. Computer Science, together with Psychology and Education, has been trying to refine teaching computational tools towards personalized self-learning [2,3, 4]. Every day, new approaches to the use of Computer and Education are bringing new perspectives to this area. The evolution of Computer and Education became computational teaching environments an excellent choice for Distance Learning, by bringing new vigor to this field of science. 
E-Education projects must take into consideration that there are different classes of users. A simple result can be: the non-cooperative, those who act in a passive way or even try to frustrate the program's objective; the cooperative, who follow orientations, but do not necessarily know where to go; and the pro-active students, who know very well their objective, and search for aid to relief the task burden [2]. Obviously this method is too cursory, especially to arts e-Education. Study about cognitive expansion of queries aims at the implementation of a knowledge-based query builder that allows complex query building in tight cooperation with the user. Due to the cognitive knowledge involved, these complex queries are expected to return more relevant results than traditional database queries or classifications [5], [6]. Besides advanced techniques in personalization (preference modeling, etc.) research issues will have to focus on results from sociology and psychology to include implicit knowledge about human behavior and social expectations in the retrieval process.

The teaching methodology employed in each case is different and there must have a clear concern by the technological environment on the profile of the user that will use the system. In order to reach this goal, cognitive student's modeling is required, and it must make a clear specification of him or hers, including his or her psychology. One main purpose of Artificial Psychology technologies is to imitate human psychology with computer, meanwhile, to provide some subjective evaluations for the objective things. This kind of research in e-Education is still at the beginning.

Section 2 implements a multi-agent architecture, based on the Java Agent framework for Distance learning Environments - JADE project, for approach to adaptive e-Education, in which a knowledge base is used to manage experimental knowledge of users. In Sections 3, the cycle of agents' adaptation, the adaptation of rules and the adaptation of psychology are illustrated respectively. The conclusions are stated in Section 4.

\section{Multi-Agent Architecture for Adaptive E-Education System}

The Java Agent framework for Distance learning Environments - JADE project [7] proposes an infrastructure of project, development and implementation of Distribute Intelligent Learning Environments - DILE, based on the approach of Multi-Agents architecture towards Distance Education, for multiple domains.

JADE architecture encompasses a Multi-Agent environment composed of an agent responsible for the system general control (Student's Model), and a Communication Manager (Manager agent) and other agents (Teacher agents), which are responsible for tasks related to their teaching tactics, where each agent may have its tasks specified according to its goal. All actions of student's data accessing are taken by the Student's Model, thus when a teacher agent is required to update the student's historic, this agent sends to the Student Model data to be updated, as well as any other change in the student's state of teaching. The tasks performed in teaching are decomposed and performed individually or in groups of agents. How the task will be decomposed is defined by the content of messages exchanged between 
agents. In our work, we aim at perceptual situations such as the e-Education of arts and put our emphases on the user satisfaction (see Fig. 1). The teacher agent dispensable and the student model agent cares more about the experimental knowledge than JADE, and one additional work of the browser agent is cognitive queries.

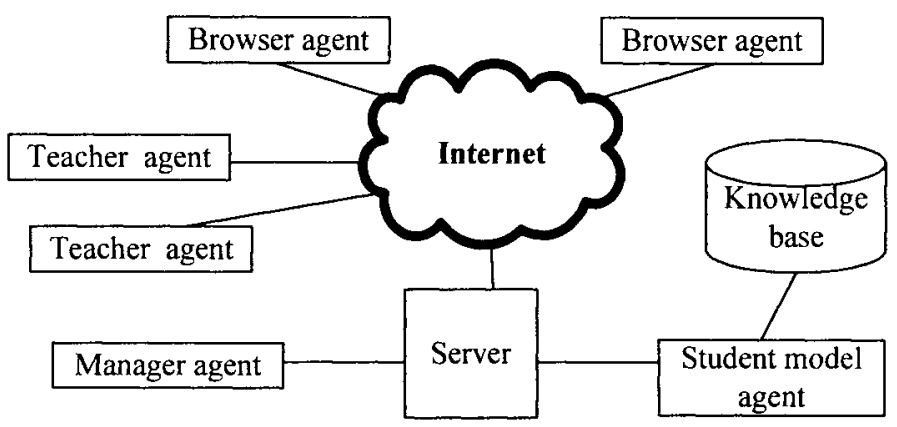

Fig.1. the multi-agent architecture for adaptive e-Education system.

The knowledge base plays an important role in adaptive e-Education systems. User psychology, a kind of implicit knowledge, is quantified in our work and a new cognitive model involving artificial psychology is presented in Fig. 2. The skills are the skills of the user it represents. It communicates by exchanging information about the knowledge base of the whole system. Beliefs are related to the description that an agent or user possesses about the others' and its own skills. And the psychology: the knowledge base should represent a student's psychology. Semantic beliefs are not enough to satisfy the user. 


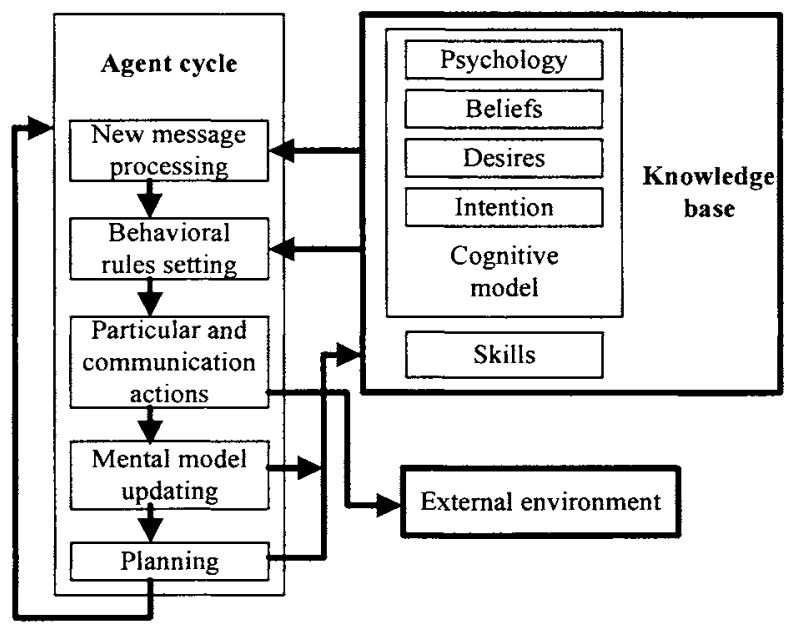

Fig. 2. The knowledge-based cycle of agents' adaptation or execution consists of five stages, and it is also a co-evolution process of the agents and the knowledge base.

\section{The Knowledge Based Adaptation}

\subsection{The Agents' Adaptation Cycle}

The cycle of agents' adaptation or execution, also shown in Fig. 2, consists of the following steps:(1) New messages processing: the task is decomposed;(2) Determination of which rules are suitable in the current situation: analysis of task and if necessary delegation of other agent(s) task;(3)Execution of actions specified for such rules: task execution;(4) Mental state update according to those rules: management of knowledge about the world, including the artificial psychology;(5) Planning: module that must develop plans that reach goals specified by agents intentions.

\subsection{The Adaptation of Rules}

The key point of this adaptive approach of e-Education is the adaptation of rules based on the adaptation of user psychology and the adaptation of beliefs, desires and intention. Figure 3 describes the determination of rules in our approach. The user profiles and the cognitive queries files built by the browser agent consists basic information about user usage and preference, from which beliefs, desires and intention can be draw as JADE does in [7]. And user's artificial psychology can also be build from them, which will appear in section 3.3. The belief technology is comparatively mature in e-Education system, so in view of both efficiency and effect, artificial psychology technology is applied to refine the suggestion results of beliefs, 
desires and intention. This process is dynamic, so the determination and the adaptation of rules are dynamic too.

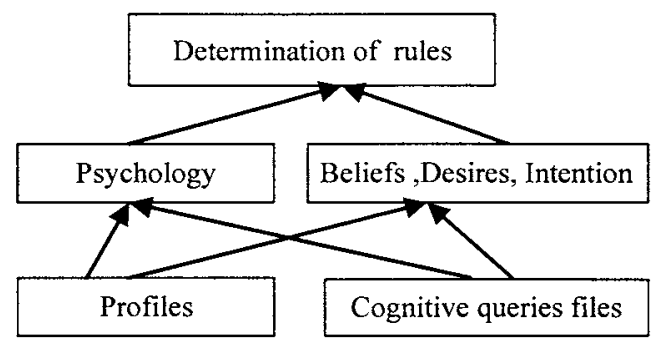

Fig. 3. Determination of rules based on the adaptation of user psychology and the adaptation of beliefs, desires and intention.

\subsection{The Adaptation of Psychology}

Aiming to maintain the Psychology, a cognitive expander is developed. Figure 4 shows the generalized architecture of cognitive expander.

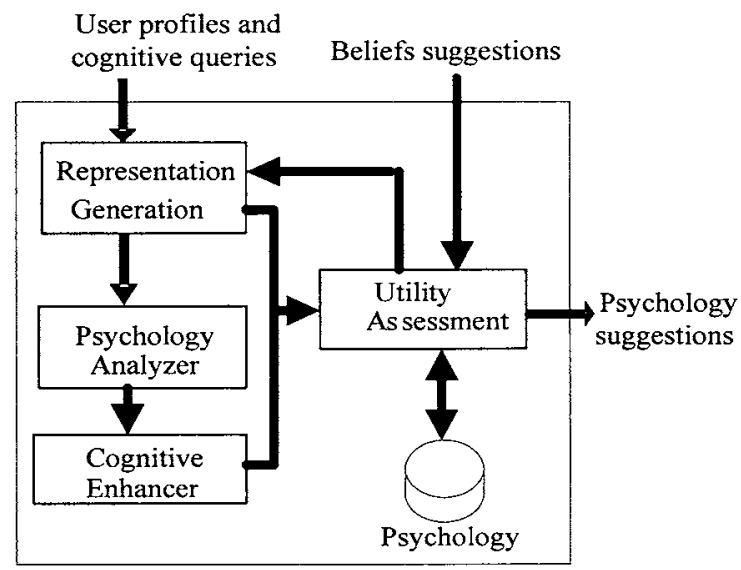

Fig.4. The cognitive expander to maintain the Psychology in the knowledge base using artificial psychology techniques.

Each user session in a user session file can be thought of in two ways; either as a single transaction of many page references, or a set of many transactions each consisting of a single page reference. The goal of transaction identification is to dynamically create meaningful clusters of references for each user. Based on an 
underlying model of the user's browsing behavior, each page reference can be categorized as a content reference, auxiliary (or navigational) reference, or hybrid. In this way different types of transactions can be obtained from the user session file. The details of methods for transaction identification are discussed in [10].

Based on the user model in [11] the user query and answer in user transaction file and user cognitive queries files is enhanced with cognitive psychology and the subjective expected utility is assigned. Considering the utilities for different combinations of preferences within a query a trade-off between query complexity or processing costs and an expected improvement of the query result has to be optimized. Comparing subjective expected utilities eventually leads to decisions about the retrieval strategy and an adequate query can be generated. This query is processed by the database retrieval system and the results are returned to the user. The essential component is the Psychology Analyzer that refines the transaction file and passes user psychology to the Cognitive Enhancer.

Cognitive expander aims at the implementation of a query builder based on psychology knowledge that allows complex query building in tight cooperation with the user accord with individual psychology preference. The Psychology Analyzer studies users' psychology using Artificial Psychology model. Cognitive Enhancer should remember the info (query and evaluation) of the former cases made by every user, and analysis the query and evaluation to gain experience about user Psychology. A repository is used to record the evaluation of former cases of one user. Psychology Analyzer use Artificial Psychology (AP) to analysis the preference of user. First, the Representation Generation collects the adjectives used by user to describe what he or she wants and whether he or she is satisfied with the service, such as "lovelybothersome", "magnificent-plain". However, not all the adjectives pairs are necessary; some adjective pairs have close correlation. So the representative pairs should be abstracted by factor analysis. The repository will record the numeric evaluation value of every case corresponding to the adjective pair. Second, the user should fill a questionnaire to describe the service that he needs, which is a new query. At last, Cognitive Enhancer will build the appropriate query.

For example, a user want to find a picture or flash in the system, firstly, there is a user preference value correspondence with the adjacent pair, and we call them average of the representative pairs, such as table 1 .

Table 1. Average of the representative pairs: user preference values correspondence with the adjacent pairs.

\begin{tabular}{|c|c|c|c|c|c|}
\hline & Case 1 & Case 2 & Case 3 & Case 4 & Case 5 \\
\hline Expensive-cheap & 0.3 & 0.6 & 0.8 & 0.1 & 0.5 \\
\hline Romantic-prim & 0.5 & 0.7 & 0.9 & 0.4 & 0.3 \\
\hline Modern-outdated & 0.1 & 0.8 & 0.3 & 0.7 & 0.2 \\
\hline$\ldots$ & $\ldots$ & $\ldots$ & $\ldots$ & $\ldots$ & $\ldots$ \\
\hline
\end{tabular}

We can get the value like this, for instance, for expensive-cheap, 0.1 represent cheapest, 0.5 represent not expensive and not cheap, 0.9 represent most expensive. Now we find out the numerical representations. 
Then we select red, green, blue, lightness, cold or warm as the items. We measure the color value (0-255) by PHOTOSHOP; the value of lightness and the value $(0-100)$ of cold or warm are obtained by the questionnaire. Table 2 gives the result:

Table 2. Characters determination and numerical representation of the samples.

\begin{tabular}{|c|c|c|c|c|c|}
\hline & sample 1 & sample 2 & sample 3 & sample 4 & sample 5 \\
\hline red & 99 & 240 & 85 & 246 & 8 \\
\hline green & 208 & 139 & 180 & 255 & 194 \\
\hline blue & 37 & 74 & 23 & 10 & 234 \\
\hline lightness & 60 & 50 & 80 & 10 & 40 \\
\hline $\begin{array}{c}\text { cold or } \\
\text { warm }\end{array}$ & 50 & 80 & 40 & 70 & 20 \\
\hline
\end{tabular}

The corresponding reactor matrix:

$$
\mathrm{X}=\left[\begin{array}{ccccc}
99 & 208 & 37 & 60 & 50 \\
240 & 139 & 74 & 50 & 80 \\
85 & 180 & 23 & 80 & 40 \\
246 & 255 & 10 & 10 & 70 \\
8 & 194 & 234 & 40 & 20
\end{array}\right]
$$

With the help of Quantification Theory I, each case can be written in the form of reactor matrix. According to the formula of Quantification Theory I :

$$
b=\left(X^{\prime} X\right)^{-1} X^{\prime} Y
$$

We obtain the quantification relationship of the adjective pairs and the case, with $\mathrm{Y}$ is a column vector and its value is the average of the previously evaluation corresponding to an adjective pair, like table 2 . $\mathrm{X}$ is the reactor matrix. All values of $\mathrm{b}$ correspond with the adjective pairs are obtained. By replacing $\mathrm{b}$ with its expression, we can calculate the evaluation $Y$ of case with the help of the predictive formula

$$
\hat{Y}=X b
$$

\[ \hat{Y}=X b \]
Then we calculate the sample multiple correlation coefficient of $\hat{Y}$ from formula 4 to checkout the validity of predictive results:

$$
r_{\hat{y} y}=\sqrt{\frac{\sum_{i=1}^{n}\left(\hat{y}_{i}-\bar{y}\right)^{2}}{\sum_{i=1}^{n}\left(y_{i}-\bar{y}\right)^{2}}}
$$

The quantification relationships $\{b\}$ are stored in the repository. When a query comes, the Cognitive Expander will depend on its repository to calculate the evaluation $Y$ to refine the suggestions of beliefs; and from the user feedback and formula 4 , the adaptation or recalculation can be trigged. Surely it is going to be more and more accurate over time. The consequence is an improving selfevolution process between users and the psychology. 


\section{Conclusions}

In this paper, advanced e-Education approach using cognitive techniques and artificial psychology techniques is investigated. Usability of e-Education has been implemented basically. Customer satisfaction has aroused concerns in these fields. Thus systems should manage experimental knowledge of users to satisfy them and support them effectively.

In the process of the model construction, we synthesize data mining techniques and AP mathematics theories, and integrate them into cognitive theory to form the Architecture for Adaptive E-Education System.

The analysis of knowledge based adaptation and Cognitive Expander applying artificial psychology technology to refine the suggestion results of beliefs, desires and intention will lead to the adaptation of agents' cycle and e-Education. And the efficiency and convenience of this system will be our future works.

\section{References}

1. A. Carmelo, "Towards Guidelines for Usability of e-Learning Applications", UI4All 2004, LNCS 3196, 185-202(2004).

2. A.S. Ricardo and M. V. Rosa, "Improving Interactivity in e-Learning Systems with Multi-agent Architecture", AH 2002, LNCS 2347, 466-471(2002).

3. Q.M. He, "Design and Implementation of a J2EE-Based Platform for Network Teaching", ICWL 2005, LNCS 3583, 49-55(2005).

4. G. Lekakos, K. Chorianopoulos, D. Spinellis, "Information systems in the living room: A case study of personalized interactive TV design", Proceedings of the 9th European Conference on Information Systems. Moderna Organizacija: Kranj, 2001.

5. D. Emmanuel, "Dinosys: An Annotation Tool for Web-Based Learning”, ICWL 2004, LNCS 3143, 59-66(2004).

6. D. Patrick and V.Q. Le, "A Question Answering Mining platform",0-7803-85969/04/2004 IEEE.

7. Silivira, A. Ricardo, Vicari and M. Rosa, "JADE - Java Agents for Distance Education Framework", In: DEC 2001, 2001, Austin. DEC 2001. CD-ROM, 2001.

8. L.N. Martin, "A Mixed XML-JavaBeans Approach to Developing T-learning Applications for the Multimedia Home Platform", MIPS 2003, LNCS 2899, 376387(2003).

9. Q. Lu, "Web Personalization Based on Artificial Psychology", WISE 2006 Workshops, LNCS 4256, 223 -229(2006).

10. B. Mobasher, H.H. Dai, T. Luo, N.A. Miki, Y.Q. Sun and W. Jim, "Discovery of Aggregate Usage Profiles for Web Personalization", Proceedings of the Web mining for E-commerce Workshop, Boston, 2000

11. R. Cooley, B. Mobasher and J. Srivastava, "Data preparation for mining World Wide Web browsing patterns",Journal of Knowledge and Information Systems, (1) 1, 1999.

12. D.S. Statelov, "SMART EDU A new TV video enabled interactive e-learning platform", 0-7803-7993-4/03 2003 IEEE. 